\title{
Positive solutions for a class of integral boundary value condition of fractional differential equations with a parameter
}

\section{Chen Yang}

Basic Course Department, Business College of Shanxi University, Taiyuan 030031, Shanxi, P. R. China.

Communicated by Y. J. Cho

\begin{abstract}
In this work, we study a class of integral boundary value condition of fractional differential equations with a parameter. The existence and uniqueness of positive solutions to the boundary value problem is established. Further, we present some properties of positive solutions to the boundary value problem dependent on the parameter. The method employed is a fixed point theorem of concave operators in partial ordering Banach spaces. As applications, two examples are given to illustrate our main results. (C)2017 All rights reserved.
\end{abstract}

Keywords: Fractional order derivative, positive solution, parameter, fixed point theorem of concave operator, integral boundary value condition.

2010 MSC: 26A33, 34B18, 34B27.

\section{Introduction}

In this article, we study the following integral boundary value condition of fractional differential equations with a parameter

$$
\left\{\begin{array}{l}
D_{0^{+}}^{\alpha} u(t)+\lambda f(t, u(t))=0, \quad 0<t<1 \\
u(0)=u^{\prime}(0)=0, \quad u(1)=\int_{0}^{1} g(s) u(s) d s
\end{array}\right.
$$

where $2<\alpha \leqslant 3, \lambda>0$ is a parameter. $\mathrm{D}_{0^{+}}^{\alpha}$ is the standard Riemann-Liouville fractional derivative of order $\alpha$ which is defined as follows:

$$
D_{0^{+}}^{\alpha} u(t)=\frac{1}{\Gamma(n-\alpha)}\left(\frac{d}{d t}\right)^{n} \int_{0}^{t}(t-s)^{n-\alpha-1} u(s) d s, \quad n=[\alpha]+1,
$$

here $\Gamma$ denotes the Euler gamma function and $[\alpha]$ denotes the integer part of number $\alpha$, provided that the right side is pointwise defined on $(0,+\infty)$, see [10].

Recently, fractional differential equations with integral boundary conditions have been extensively studied. For a small sample of such work, we refer the reader to $[1-3,5,7,9,11,17,18]$ and the references

Email address: yangchen0809@126.com (Chen Yang)

doi:10.22436/jnsa.010.05.37 
therein. As it has been stated in [3], integral boundary value problems have many various applications in applied fields such as blood flow problems, chemical engineering, thermo-elasticity, underground water flow and population dynamics. The existence of solutions for integral boundary value problems is an important problem. In literature, most of the authors have studied the existence and multiplicity of positive solutions. Recently, in paper [11], the authors considered a class of fractional equations involving the Riemann-Liouville fractional derivative with integral boundary value conditions

$$
\left\{\begin{array}{l}
D_{0^{+}}^{\alpha} u(t)+q(t) f(t, u(t))=0, \quad 0<t<1 \\
u(0)=u^{\prime}(0)=0, \quad u(1)=\int_{0}^{1} g(s) u(s) d s
\end{array}\right.
$$

where $2<\alpha \leqslant 3, q, g \in \mathrm{L}^{1}[0,1]$. The authors obtained some properties of Green function of this problem and by using monotone iteration method, established the existence of one positive solution for the problem (1.2). But the uniqueness of positive solutions is not treated in [11]. From literature, we know that the existence and uniqueness results of positive solutions for fractional differential equations with integral boundary conditions are still very few. Moreover, there are also few papers reported on integral boundary conditions of fractional differential equations with a parameter. So it is important to bridge this gap between known integer-order boundary value problems studies and unknown fractional boundary value problems theory.

Inspired by the work [11] and many known results in [12, 14-16], we will study the problem (1.1) with concave nonlinearity. Different from the works mentioned above, in this paper we will use a fixed point theorem of concave operators in partial ordering Banach spaces to show the existence and uniqueness of positive solutions for the problem (1.1). Moreover, we present some good properties of positive solutions to the boundary value problem dependent on the parameter. The methods used here are different from those in previous articles.

In this paper, our basic assumptions on functions $f(t, x), g(t)$ here are:

$\left(\mathrm{H}_{1}\right) \mathrm{f}:[0,1] \times[0,+\infty) \rightarrow[0,+\infty)$ is continuous;

$\left(\mathrm{H}_{2}\right) \mathrm{f}(\mathrm{t}, \mathrm{x})$ is concave in $\mathrm{x}$ for each $\mathrm{t} \in[0,1]$;

$\left(\mathrm{H}_{3}\right) \mathrm{g}:[0,1] \rightarrow[0,+\infty)$ with $\mathrm{g} \in \mathrm{L}^{1}[0,1]$ and $\delta:=\int_{0}^{1} \mathrm{~s}^{\alpha-1}(1-\mathrm{s}) \mathrm{g}(\mathrm{s}) \mathrm{d} s>0, \sigma:=\int_{0}^{1} \mathrm{~s}^{\alpha-1} \mathrm{~g}(\mathrm{~s}) \mathrm{d} s<1$;

$\left(\mathrm{H}_{4}\right)$ there exist constants $a, b>0$ such that $f(t, 0) \geqslant a, f(t, 1) \leqslant b$, for all $t \in[0,1]$;

$\left(\mathrm{H}_{5}\right) \lim _{x \rightarrow \infty} \frac{f(t, x)}{x}=0$ uniformly on $[0,1]$.

Remark 1.1. From $\left(\mathrm{H}_{2}\right)$, we know that $\mathrm{f}(\mathrm{t}, \mathrm{x})$ is increasing in $x \in[0,+\infty)$.

\section{Preliminaries}

In the following, we list some basic concepts in ordered Banach spaces and a fixed point theorem which will be used later. For convenience of readers, we suggest that one refer to $[4,6,8]$ for details.

Let $(E,\|\cdot\|)$ be a real Banach space, $\theta$ is the zero element of $E$. A non-empty set $P \subset E$ is a cone. Then $E$ is partially ordered by $P$, i.e., $x \leqslant y$ if and only if $y-x \in P$. If $x \leqslant y$ and $x \neq y$, then we denote $x<y$ or $y>x$. Set $\stackrel{\circ}{P}=\{x \in P \mid x$ is an interior point of $P\}$, a cone $P$ is called to be solid if $\stackrel{\circ}{P}$ is non-empty. The

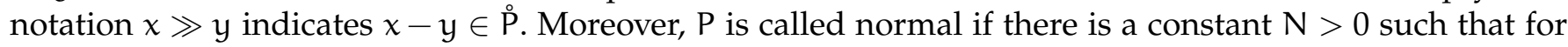
all $x, y \in E, \theta \leqslant x \leqslant y$ implies $\|x\| \leqslant N\|y\|$; in this case $N$ is the infimum of such constants, it is called the normality constant of $P$.

Definition 2.1. Assume $D$ is a convex subset in $E$. An operator $A: D \rightarrow E$ is called a concave operator if $A(t x+(1-t) y) \geqslant t A x+(1-t) A y$, for $x, y \in D$ and $t \in[0,1]$.

Definition 2.2. Let $P$ be a cone in $E$ and $e \in P \backslash \theta$, set $E_{e}=\{x \in E$ : there exists $\lambda>0$ such that $-\lambda e \leqslant x \leqslant \lambda e\}$, and $\|x\|_{e}=\inf \{\lambda>0:-\lambda e \leqslant x \leqslant \lambda e\}$, for all $x \in E_{e}$. Then $E_{e}$ becomes a normed linear space under the norm $\|\cdot\|_{e}$, and $\|x\|_{e}$ is called the e-norm of the element $x \in E_{e}$. 
Lemma 2.3 ([6, Theorem 1.5.1]). Assume cone P is normal. Then the following conclusions hold:

(i) $\mathrm{E}_{e}$ is a Banach space with e-norm, and there exists a constant $\mathrm{l}>0$ such that $\|\mathrm{x}\| \leqslant \mathrm{l}\|\mathrm{x}\|_{e}$, for all $\mathrm{x} \in \mathrm{E}_{e}$;

(ii) $P_{e}=E_{e} \cap P$ is a normal solid cone in $E_{e}$, and $P_{e}^{\circ}=\left\{x \in E_{e}\right.$ : there exists $\tau=\tau(x)>0$ such that $\left.x \geqslant \tau e\right\}$.

Lemma 2.4 ([18]). Assume $\left(\mathrm{H}_{3}\right)$ holds. Let $2<\alpha \leqslant 3$ and $\mathrm{y} \in \mathrm{C}[0,1]$, then the unique solution of the following boundary value problem

$$
\left\{\begin{array}{l}
D_{0^{+}}^{\alpha} u(t)+y(t)=0, \quad 0<t<1 \\
u(0)=u^{\prime}(0)=0, \quad u(1)=\int_{0}^{1} g(s) u(s) d s
\end{array}\right.
$$

is given by

$$
u(t)=\int_{0}^{1} G(t, s) y(s) d s, \quad t \in[0,1]
$$

where

$$
\begin{aligned}
G(t, s) & =G_{1}(t, s)+G_{2}(t, s), \quad(t, s) \in[0,1] \times[0,1], \\
G_{1}(t, s) & =\frac{1}{\Gamma(\alpha)}\left\{\begin{array}{l}
t^{\alpha-1}(1-s)^{\alpha-1}-(t-s)^{\alpha-1}, \quad 0 \leqslant s \leqslant t \leqslant 1, \\
t^{\alpha-1}(1-s)^{\alpha-1}, \quad 0 \leqslant t \leqslant s \leqslant 1,
\end{array}\right. \\
G_{2}(t, s) & =\frac{t^{\alpha-1}}{1-\sigma} \int_{0}^{1} G_{1}(\tau, s) g(\tau) d \tau .
\end{aligned}
$$

Lemma 2.5 ([13]). The function $\mathrm{G}_{1}(\mathrm{t}, \mathrm{s})$ given in Lemma 2.4 has the following properties:

$$
\frac{t^{\alpha-1}(1-t) s(1-s)^{\alpha-1}}{\Gamma(\alpha)} \leqslant G_{1}(t, s) \leqslant \frac{s(1-s)^{\alpha-1}}{\Gamma(\alpha-1)}, \quad \forall t, s \in[0,1]
$$

Lemma 2.6. The Green function $\mathrm{G}(\mathrm{t}, \mathrm{s})$ given in Lemma 2.4 satisfies the inequalities

$$
\frac{\delta s(1-s)^{\alpha-1} t^{\alpha-1}}{(1-\sigma) \Gamma(\alpha)} \leqslant G(t, s) \leqslant \frac{t^{\alpha-1}(1-s)^{\alpha-1}}{(1-\sigma) \Gamma(\alpha)}, \quad t, s \in[0,1]
$$

Proof. From Lemma 2.4 in [11], the right inequality holds. So we only need to prove the left inequality. From Lemma 2.5, we obtain

$$
\begin{aligned}
G(t, s) & =G_{1}(t, s)+G_{2}(t, s) \geqslant G_{2}(t, s)=\frac{t^{\alpha-1}}{1-\sigma} \int_{0}^{1} G_{1}(\tau, s) g(\tau) d \tau \\
& \geqslant \frac{t^{\alpha-1}}{1-\sigma} \int_{0}^{1} \frac{\tau^{\alpha-1}(1-\tau) s(1-s)^{\alpha-1}}{\Gamma(\alpha)} g(\tau) d \tau \\
& =\frac{t^{\alpha-1} s(1-s)^{\alpha-1}}{(1-\sigma) \Gamma(\alpha)} \int_{0}^{1} \tau^{\alpha-1}(1-\tau) g(\tau) d \tau \\
& =\frac{\delta s(1-s)^{\alpha-1} t^{\alpha-1}}{(1-\sigma) \Gamma(\alpha)} .
\end{aligned}
$$

\section{Existence, uniqueness and properties of positive solutions for the problem (1.1)}

In this section, we apply the following theorem to study the problem (1.1).

Lemma 3.1 ([4]). Let $\mathrm{P}$ be a normal solid cone and $\mathrm{A}: \mathrm{P} \rightarrow \mathrm{P}$ be a concave operator. Assume that $\mathrm{A} \theta \gg \theta$. Then the following conclusions hold: 
(i) there exists $0<\lambda^{*} \leqslant \infty$ such that the operator equation

$$
u=\lambda A u,
$$

has a unique solution $\mathrm{u}(\lambda)$ in $\mathrm{P}$ for $0 \leqslant \lambda<\lambda^{*}$; when $\lambda \geqslant \lambda^{*},(3.1)$ has no solution in $\mathrm{P}$;

(ii) for any $\mathfrak{u}_{0} \in P$, set $\mathfrak{u}_{0}(\lambda)=u_{0}, u_{n}(\lambda)=\lambda A u_{n-1}(\lambda), n=1,2,3, \ldots$. Then, for $0<\lambda<\lambda^{*}$ we have $u_{n}(\lambda) \rightarrow u(\lambda)$ as $n \rightarrow \infty$;

(iii) $u(\cdot):\left[0, \lambda^{*}\right) \rightarrow P$ is continuous, and strongly increasing (i.e., $0 \leqslant \lambda_{1}<\lambda_{2}<\lambda^{*} \Longrightarrow u\left(\lambda_{1}\right) \ll u\left(\lambda_{2}\right)$ ). Moreover, $\mathrm{u}(\mathrm{t} \lambda) \leqslant \mathrm{tu}(\lambda)$ for $0 \leqslant \lambda<\lambda^{*}, 0 \leqslant \mathrm{t} \leqslant 1$;

(iv) $\lambda \rightarrow \lambda^{*}-0$ implies $\|\mathrm{u}(\lambda)\| \rightarrow \infty$;

(v) if there exist $v_{0} \in \mathrm{P}$ and $\lambda_{0}>0$ such that $\lambda_{0} A v_{0} \leqslant v_{0}$, then $\lambda^{*}>\lambda_{0}$.

Remark 3.2. If $A: P \rightarrow P$ is concave, then $A$ is increasing (see [4]).

Throughout our following considerations, we will work in $E=C[0,1]$, a Banach space with endowed with the norm $\|u\|_{E}=\max _{t \in[0,1]}|u(t)|$. Let $P=\{u \in C[0,1], u(t) \geqslant 0, \forall t \in[0,1]\}$, then $P$ is a normal solid cone of $E$. Denote $e(t)=t^{\alpha-1}, t \in[0,1]$. Then $e \in C[0,1], 0 \leqslant e(t) \leqslant 1, e(t) \not \equiv 0$. We get $e \in P \backslash\{\theta\}$.

Let $X=E_{e}=\{u \in E: \exists \tau>0$, s.t. $-\tau e(t) \leqslant u(t) \leqslant \tau e(t), \forall t \in[0,1]\}$, endowed with the norm $\|u\|_{X}=\inf \{\tau>0:-\tau e(t) \leqslant u(t) \leqslant \tau e(t), \forall t \in[0,1]\}$. Set $\widetilde{P}=X \cap P$. From Lemma 2.3, we know that $X$ is a Banach space, $\widetilde{P}$ is a normal solid cone of $X$ and $\stackrel{\stackrel{P}{P}}{=}=\{u \in X$ : there exists $\zeta>0$ such that $u(t) \geqslant \zeta e(t), \forall t \in[0,1]\}$. In addition, there exists a constant $l>0$ such that $\|u\|_{E} \leqslant l\|u\|_{X}$, for all $u \in X$.

Theorem 3.3. Assume $\left(\mathrm{H}_{1}\right)-\left(\mathrm{H}_{4}\right)$ hold. Then the following conclusions hold:

(i) there exists $0<\lambda^{*} \leqslant \infty$ such that, for any given $\lambda \in\left(0, \lambda^{*}\right)$, the problem (1.1) has a unique positive solution $u_{\lambda}$ in $\widetilde{\mathrm{P}}$; when $\lambda \geqslant \lambda^{*}$, the problem (1.1) has no positive solution in $\widetilde{\mathrm{P}}$;

(ii) for any $\mathrm{u}_{0} \in \widetilde{\mathrm{P}}$ and for any given $\lambda \in\left(0, \lambda^{*}\right)$, constructing successively the sequence

$$
u_{m}(t)=\lambda \int_{0}^{1} G(t, s) f\left(s, u_{m-1}(s)\right) d s, \quad m=1,2,3, \cdots,
$$

then we have

$$
\max _{t \in[0,1]}\left|u_{m}(t)-u_{\lambda}(t)\right| \rightarrow 0 \text { as } m \rightarrow \infty ;
$$

(iii) $\max _{\mathfrak{t} \in[0,1]}\left|\mathfrak{u}_{\lambda}(\mathfrak{t})-\mathfrak{u}_{\lambda_{0}}(\mathfrak{t})\right| \rightarrow 0$ as $\lambda \rightarrow \lambda_{0}$ where $0<\lambda_{0}<\lambda^{*}$, and if $0<\lambda_{1}<\lambda_{2}<\lambda^{*}$ then

$$
u_{\lambda_{1}}(t) \leqslant u_{\lambda_{2}}(t), \quad \forall t \in[0,1]
$$

and $u_{\lambda_{1}}(t) \not \equiv u_{\lambda_{2}}(t)$

(iv) $u_{r \lambda}(t) \leqslant r u_{\lambda}(t), r, t \in[0,1]$ where $\lambda \in\left(0, \lambda^{*}\right)$.

Proof. From Lemma 2.4, we know that $u(t)$ is the solution of the problem (1.1), if and only if

$$
u(t)=\lambda \int_{0}^{1} G(t, s) f(s, u(s)) d s,
$$

where $G(t, s)$ is given as in Lemma 2.4. Define an operator $A$ by

$$
(A u)(t)=\int_{0}^{1} G(t, s) f(s, u(s)) d s .
$$


It is easy to prove that $u$ is the solution of the problem (1.1), if and only if $u=\lambda A u$. For $u \in P$, in view of the continuity of functions $G(t, s)$ and $f(t, x)$, we have $A u \in C[0,1]$. And from $G(t, s) \geqslant 0$ and $f(t, x)>0$, for all $t \in[0,1]$, we can get $(A u)(t) \geqslant 0, t \in[0,1]$. So $A u \in P$. Since $f(t, \cdot)$ is concave, for $x>1$, we have

$$
f(t, 1)=f\left(t, \frac{1}{x} \cdot x+\left(1-\frac{1}{x}\right) \cdot 0\right) \geqslant \frac{1}{x} f(t, x)+\left(1-\frac{1}{x}\right) f(t, 0),
$$

and thus from $\left(\mathrm{H}_{4}\right)$, we get

$$
f(t, x) \leqslant x f(t, 1)-(x-1) f(t, 0) \leqslant x f(t, 1) \leqslant b x .
$$

Therefore, for $u \in P$, from Lemma 2.6 we have

$$
\begin{aligned}
0 & \leqslant \int_{0}^{1} G(t, s) f(s, u(s)) d s \leqslant \int_{0}^{1} \frac{t^{\alpha-1}(1-s)^{\alpha-1}}{(1-\sigma) \Gamma(\alpha)} f\left(s,\|u\|_{E}\right) d s \\
& \leqslant \frac{t^{\alpha-1}}{(1-\sigma) \Gamma(\alpha)} \int_{0}^{1}(1-s)^{\alpha-1} f\left(s,\|u\|_{E}+1\right) d s \\
& \leqslant \frac{t^{\alpha-1}}{(1-\sigma) \Gamma(\alpha)} \int_{0}^{1}(1-s)^{\alpha-1} b\left(1+\|u\|_{E}\right) d s \\
& =\frac{b\left(1+\|u\|_{E}\right)}{(1-\sigma) \alpha \Gamma(\alpha)} t^{\alpha-1}=\frac{b\left(1+\|u\|_{E}\right)}{(1-\sigma) \alpha \Gamma(\alpha)} e(t) \\
& =M_{1} e(t), \quad t \in[0,1],
\end{aligned}
$$

where $M_{1}=\frac{b\left(1+\|u\|_{E}\right)}{(1-\sigma) \alpha \Gamma(\alpha)}>0$. Thus, $0 \leqslant(A u)(t) \leqslant M_{1} e(t)$, so $A u \in X$. Then $A u \in P \cap X$, i.e., $A u \in \widetilde{P}$.

Hence, we obtain $A: P \rightarrow \widetilde{P}$, and thus $A: \widetilde{P} \rightarrow \widetilde{P}$. Further, from $f(t, \cdot)$ is concave, we can see that operator $A$ is concave. By Lemma 2.6 and $\left(\mathrm{H}_{4}\right)$,

$$
\begin{aligned}
A \theta(t) & =\int_{0}^{1} G(t, s) f(s, 0) d s \geqslant \int_{0}^{1} \frac{\delta s(1-s)^{\alpha-1} t^{\alpha-1}}{(1-\sigma) \Gamma(\alpha)} a d s \\
& =\frac{a \delta t^{\alpha-1}}{(1-\sigma) \Gamma(\alpha)} \int_{0}^{1} s(1-s)^{\alpha-1} d s=\frac{a \delta}{(1-\sigma) \alpha(\alpha+1) \Gamma(\alpha)} e(t), \quad t \in[0,1] .
\end{aligned}
$$

Since $\frac{a \delta}{(1-\sigma) \alpha(\alpha+1) \Gamma(\alpha)}>0$, we have $A \theta \in \stackrel{\circ}{\widetilde{P}}$, i.e., $A \theta \gg \theta$. From Lemma 3.1, there exists $0<\lambda^{*} \leqslant \infty$ such that the operator equation $u=\lambda A u$ has a unique solution $u_{\lambda}$ in $\widetilde{P}$ for $0<\lambda<\lambda^{*}$. When $\lambda \geqslant \lambda^{*}, u=\lambda A u$ has no solution in $\widetilde{P}$. For any $u_{0} \in \widetilde{P}$, set $u_{m}=\lambda A u_{m-1}, m=1,2, \cdots$, then for $0<\lambda<\lambda^{*}$, we have $u_{m} \rightarrow u_{\lambda}$ as $m \rightarrow \infty$. $u_{\lambda}:\left(0, \lambda^{*}\right) \rightarrow \widetilde{P}$ is continuous, and strongly increasing (i.e., $0<\lambda_{1}<\lambda_{2}<\lambda^{*} \Longrightarrow$ $\left.u_{\lambda_{1}} \ll u_{\lambda_{2}}\right)$. Moreover, $u_{(t \lambda)} \leqslant t u_{\lambda}$ for $0 \leqslant \lambda<\lambda^{*}, 0 \leqslant t \leqslant 1, \lambda \rightarrow \lambda^{*}-0$ implies $\left\|u_{\lambda}\right\|_{x} \rightarrow \infty$. That is,

(i) for any given $\lambda \in\left(0, \lambda^{*}\right)$, the problem (1.1) has a unique positive solution $u_{\lambda}$ in $\widetilde{P}$; when $\lambda \geqslant \lambda^{*}$, the problem (1.1) has no positive solution in $\widetilde{\mathrm{P}}$;

(ii) for any $u_{0} \in \widetilde{P}$ and for any given $\lambda \in\left(0, \lambda^{*}\right)$, constructing successively the sequence

$$
u_{m}(t)=\lambda \int_{0}^{1} G(t, s) f\left(s, u_{m-1}(s)\right) d s, \quad m=1,2, \cdots,
$$

then

$$
\max _{t \in[0,1]}\left|u_{m}(t)-u_{\lambda}(t)\right| \rightarrow 0 \text { as } m \rightarrow \infty
$$

(iii) $\max _{t \in[0,1]}\left|\mathfrak{u}_{\lambda}(t)-\mathfrak{u}_{\lambda_{0}}(t)\right| \rightarrow 0$ as $\lambda \rightarrow \lambda_{0}$, where $0<\lambda_{0}<\lambda^{*}$, and if $0<\lambda_{1}<\lambda_{2}<\lambda^{*}$, then there exists $\tau>0$ such that $u_{\lambda_{2}}(t)-u_{\lambda_{1}}(t) \geqslant \tau e(t), t \in[0,1]$ and thus $u_{\lambda_{1}}(t) \leqslant u_{\lambda_{2}}(t)$, for all $t \in[0,1]$ and $u_{\lambda_{1}}(t) \not \equiv u_{\lambda_{2}}(t)$; 
(iv) $u_{r \lambda}(t) \leqslant r u_{\lambda}(t), r, t \in[0,1]$, where $\lambda \in\left(0, \lambda^{*}\right)$.

So the conclusions (i), (ii), (iii), and (iv) hold.

Theorem 3.4. Assume $\left(\mathrm{H}_{1}\right)-\left(\mathrm{H}_{5}\right)$ hold. Then the following conclusions hold:

(i) for any given $\lambda>0$, the problem (1.1) has a unique positive solution $\mathrm{u}_{\lambda}$ in $\widetilde{\mathrm{P}}$;

(ii) for any $\mathrm{u}_{0} \in \widetilde{\mathrm{P}}$ and for any given $\lambda>0$, constructing successively the sequence

$$
u_{m}(t)=\lambda \int_{0}^{1} G(t, s) f\left(s, u_{m-1}(s)\right) d s, \quad m=1,2,3, \cdots,
$$

then we have

$$
\max _{t \in[0,1]}\left|u_{m}(t)-u_{\lambda}(t)\right| \rightarrow 0 \text { as } \mathrm{m} \rightarrow \infty
$$

(iii) $\max _{\mathfrak{t} \in[0,1]}\left|\mathrm{u}_{\lambda}(\mathrm{t})-\mathrm{u}_{\lambda_{0}}(\mathrm{t})\right| \rightarrow 0$ as $\lambda \rightarrow \lambda_{0}$ where $0<\lambda_{0}$, and if $0<\lambda_{1}<\lambda_{2}$, then $\mathrm{u}_{\lambda_{1}}(\mathrm{t}) \leqslant \mathrm{u}_{\lambda_{2}}(\mathrm{t})$, for all $\mathrm{t} \in[0,1]$ and $\mathrm{u}_{\lambda_{1}}(\mathrm{t}) \not \equiv \mathfrak{u}_{\lambda_{2}}(\mathrm{t})$;

(iv) $u_{r \lambda}(t) \leqslant r u_{\lambda}(t), r, t \in[0,1]$ where $\lambda>0$.

Proof. From the proof of Theorem 3.3, we only need to prove $\lambda^{*}=\infty$ for any $\lambda>0$ is given. From $\left(\mathrm{H}_{5}\right)$, we can take a $T>0$ large enough such that $f(t, T) \leqslant \lambda^{-1}(1-\sigma) \alpha \Gamma(\alpha) T$, for all $t \in[0,1]$. Set $v_{0}(t)=T e(t)$, so

$$
\begin{aligned}
\lambda\left(A v_{0}\right)(t) & =\lambda \int_{0}^{1} G(t, s) f(s, T e(s)) d s \leqslant \lambda \int_{0}^{1} G(t, s) f(s, T) d s \\
& \leqslant \lambda \int_{0}^{1} \frac{(1-s)^{\alpha-1}}{(1-\sigma) \Gamma(\alpha)} f(s, T) d s \cdot t^{\alpha-1} \\
& \leqslant \lambda \frac{t^{\alpha-1}}{(1-\sigma) \Gamma(\alpha)} \lambda^{-1}(1-\sigma) \alpha \Gamma(\alpha) T \int_{0}^{1}(1-s)^{\alpha-1} d s \\
& =T e(t)=v_{0}(t) \\
v_{0}(t)-\lambda\left(A v_{0}\right)(t) & =T e(t)-\lambda \int_{0}^{1} G(t, s) f(s, T e(t)) d s \\
& \leqslant T e(t)+\lambda \int_{0}^{1} G(t, s) f(s, T e(t)) d s \\
& \leqslant T e(t)+\lambda \int_{0}^{1} \frac{(1-s)^{\alpha-1}}{(1-\sigma) \Gamma(\alpha)} f(s, T) d s \cdot e(t) \\
& \leqslant\left[T+\lambda \int_{0}^{1} \frac{b(1+T)}{(1-\sigma) \Gamma(\alpha)}(1-s)^{\alpha-1} d s\right] e(t) \\
& =\left[T+\frac{b \lambda(1+T)}{(1-\sigma) \alpha \Gamma(\alpha)}\right] e(t), t \in[0,1] .
\end{aligned}
$$

Therefore, $v_{0}-\lambda A v_{0} \in \widetilde{\mathrm{P}}$. That is, $\lambda A v_{0}(\mathrm{t}) \leqslant v_{0}$. From Lemma $3.1(\mathrm{v})$, we have $\lambda^{*}>\lambda$. Because $\lambda$ is arbitrary, we get $\lambda^{*}=\infty$.

To illustrate how our main results can be used in practice we present two examples. 
Example 3.5. Consider the following integral boundary value condition of fractional differential equations with a parameter

$$
\left\{\begin{array}{l}
D_{0^{+}}^{\frac{7}{3}} u(t)+\lambda(1+\sin t)\left[\arctan u(t)+\frac{\pi}{4}\right]=0, \quad t \in(0,1), \\
u(0)=u^{\prime}(0)=0, \quad u(1)=\int_{0}^{1} s^{\frac{2}{3}} u(s) d s .
\end{array}\right.
$$

In this example, $\alpha=\frac{7}{3}, \lambda>0$ is a parameter and

$$
g(t)=t^{\frac{2}{3}}, f(t, x)=(1+\sin t)\left(\arctan x+\frac{\pi}{4}\right), \quad \forall t \in[0,1] .
$$

Then we have $\delta=\int_{0}^{1} s^{\frac{4}{3}}(1-s) s^{\frac{2}{3}} d s=\frac{1}{12}>0, \sigma=\int_{0}^{1} s^{\frac{4}{3}} s^{\frac{2}{3}} d s=\frac{1}{3}<1$. Evidently, $f(t, x):[0,1] \times[0,+\infty) \rightarrow$ $[0,+\infty)$ is continuous, $f(t, \cdot)$ is increasing and concave. Take $a=\frac{\pi}{4}, b=\pi$, then

$$
f(t, 0)=(1+\sin t)\left(0+\frac{\pi}{4}\right) \geqslant \frac{\pi}{4}=a, \quad f(t, 1)=(1+\sin t)\left(\frac{\pi}{4}+\frac{\pi}{4}\right) \leqslant \pi=b .
$$

Hence, all the conditions of Theorem 3.3 are satisfied. So we have the following conclusions:

(i) there exists $0<\lambda^{*} \leqslant \infty$ such that, for any given $\lambda \in\left(0, \lambda^{*}\right)$, the problem (3.2) has a unique positive solution $u_{\lambda}$ in $\widetilde{P}$; when $\lambda \geqslant \lambda^{*}$, the problem (3.2) has no positive solution in $\widetilde{P}$, here

$$
\widetilde{P}=\left\{u \in C[0,1]: \exists \tau>0, \text { s.t. } 0 \leqslant u(t) \leqslant \tau t^{\frac{4}{3}}, \forall t \in[0,1]\right\} ;
$$

(ii) for any $\mathrm{u}_{0} \in \widetilde{\mathrm{P}}$ and for any given $\lambda \in\left(0, \lambda^{*}\right)$, constructing successively the sequence

$$
u_{m}(t)=\lambda \int_{0}^{1} G(t, s)(1+\sin s)\left[\arctan u_{m-1}(s)+\frac{\pi}{4}\right] d s, \quad m=1,2,3, \cdots,
$$

then we have

$$
\max _{t \in[0,1]}\left|u_{m}(t)-u_{\lambda}(t)\right| \rightarrow 0 \text { as } m \rightarrow \infty ;
$$

(iii) $\max _{\mathfrak{t} \in[0,1]}\left|\mathfrak{u}_{\lambda}(\mathrm{t})-\mathfrak{u}_{\lambda_{0}}(\mathrm{t})\right| \rightarrow 0$ as $\lambda \rightarrow \lambda_{0}$, where $0<\lambda_{0}<\lambda^{*}$, and if $0<\lambda_{1}<\lambda_{2}<\lambda^{*}$, then

$$
u_{\lambda_{1}}(t) \leqslant u_{\lambda_{2}}(t), \quad \forall t \in[0,1]
$$

and $u_{\lambda_{1}}(t) \not \equiv u_{\lambda_{2}}(t) ;$

(iv) $u_{r \lambda}(t) \leqslant r u_{\lambda}(t), r, t \in[0,1]$, where $\lambda \in\left(0, \lambda^{*}\right)$.

Example 3.6. Consider the following integral boundary value condition of fractional differential equations with a parameter

$$
\left\{\begin{array}{l}
D_{0^{+}}^{2.5} u(t)+\lambda\left\{\sum_{i=1}^{n_{0}} a_{i}(t)[u(t)]^{\frac{1}{i+1}}+h(t)\right\}=0, \quad t \in(0,1), \\
u(0)=u^{\prime}(0)=0, \quad u(1)=\int_{0}^{1} s u(s) d s .
\end{array}\right.
$$

In this problem, $\alpha=2.5, \lambda>0, \mathrm{n}_{0} \geqslant 1$ is an integer and

$$
g(t)=t, f(t, x)=\sum_{i=1}^{n_{0}} a_{i}(t) x^{\frac{1}{i+1}}+h(t), \quad \forall t \in[0,1],
$$

where $a_{i}(t)$ is continuous and nonnegative $\left(i=1,2, \cdots, n_{0}\right)$ and $h:[0,1] \rightarrow(0,+\infty)$ is continuous and nondecreasing. Then we have $\delta=\int_{0}^{1} s^{\alpha}(1-\mathrm{s}) \mathrm{d} s=\frac{4}{63}>0, \sigma=\int_{0}^{1} \mathrm{~s}^{\alpha} \mathrm{d} s=\frac{2}{7}<1$. Evidently, 
$f(t, x):[0,1] \times[0,+\infty) \rightarrow[0,+\infty)$ is continuous, $f(t, \cdot)$ is increasing and concave. Take $a \in(0, h(0)), b \geqslant$ $\sum_{i=1}^{n_{0}}\left\|a_{i}\right\|+h(1)$, then

$$
f(t, 0)=h(t) \geqslant h(0) \geqslant a, \quad f(t, 1)=\sum_{i=1}^{n_{0}} a_{i}(t)+h(t) \leqslant \sum_{i=1}^{n_{0}}\left\|a_{i}\right\|+h(1) \leqslant b .
$$

Further, it is easy to prove that $\lim _{x \rightarrow \infty} \frac{f(t, x)}{x}=0$ uniformly on $[0,1]$, therefore from Theorem $3.4, \lambda^{*}=\infty$. Hence, all the conditions of Theorem 3.4 are satisfied. So we have the following conclusions:

(i) for any given $\lambda \in(0,+\infty)$, the problem (3.3) has a unique positive solution $u_{\lambda}$ in $\widetilde{P}$, here

$$
\widetilde{P}=\left\{u \in C[0,1]: \exists \tau>0, \text { s.t. } 0 \leqslant u(t) \leqslant \tau t^{\frac{3}{2}}, \forall t \in[0,1]\right\} ;
$$

(ii) for any $\mathrm{u}_{0} \in \widetilde{\mathrm{P}}$ and for any given $\lambda \in(0,+\infty)$, constructing successively the sequence

$$
u_{m}(t)=\lambda \int_{0}^{1} G(t, s)\left\{\sum_{i=1}^{n_{0}} a_{i}(s)\left[u_{m-1}(s)\right]^{\frac{1}{i+1}}+h(s)\right\} d s, \quad m=1,2,3, \cdots,
$$

then we have

$$
\max _{t \in[0,1]}\left|u_{m}(t)-u_{\lambda}(t)\right| \rightarrow 0 \text { as } m \rightarrow \infty
$$

(iii) $\max _{\mathfrak{t} \in[0,1]}\left|\mathfrak{u}_{\lambda}(\mathrm{t})-\mathfrak{u}_{\lambda_{0}}(t)\right| \rightarrow 0$ as $\lambda \rightarrow \lambda_{0}$, where $0<\lambda_{0}<+\infty$, and if $0<\lambda_{1}<\lambda_{2}<+\infty$, then

$$
\mathrm{u}_{\lambda_{1}}(\mathrm{t}) \leqslant \mathrm{u}_{\lambda_{2}}(\mathrm{t}), \quad \forall \mathrm{t} \in[0,1]
$$

and $u_{\lambda_{1}}(t) \not \equiv u_{\lambda_{2}}(t) ;$

(iv) $u_{r \lambda}(t) \leqslant r u_{\lambda}(t), r, t \in[0,1]$, where $\lambda \in(0,+\infty)$.

\section{Conclusions}

In this work, we study a class of integral boundary value problem with a parameter. We obtain some new results on the existence and uniqueness of positive solutions. Moreover, we establish some good properties of positive solutions to the integral boundary value problem dependent on the parameter. The method used here is different from the literature and so is the existence and uniqueness results to integral boundary value conditions of fractional differential equations.

\section{Acknowledgment}

This paper was supported financially by the Science Foundation of Shanxi Province (2013011003-3), the Youth Science Foundation of China (11201272).

\section{References}

[1] B. Ahmad, S. Sivasundaram, Existence of solutions for impulsive integral boundary value problems of fractional order, Nonlinear Anal. Hybrid Syst., 4 (2010), 134-141. 1

[2] A. Cabada, Z. Hamdi, Nonlinear fractional differential equations with integral boundary value conditions, Appl. Math. Comput., 228 (2014), 251-257.

[3] A. Cabada, G.-T. Wang, Positive solutions of nonlinear fractional differential equations with integral boundary value conditions, J. Math. Anal. Appl., 389 (2012), 403-411. 1 
[4] Y. Du, Fixed points of a class of noncompact operator and its application, (in Chinese), Acta Mathematica Sinica, 32 (1989), 618-627. 2, 3.1, 3.2

[5] M.-Q. Feng, X.-M. Zhang, W.-G. Ge, New existence results for higher-order nonlinear fractional differential equation with integral boundary conditions, Bound. Value Probl., 2011 (2011), 20 pages. 1

[6] D. J. Guo, V. Lakshmikantham, Nonlinear problems in abstract cones, Notes and Reports in Mathematics in Science and Engineering, Academic Press, Inc., Boston, MA, (1988). 2, 2.3

[7] M. Jiang, S.-M. Zhong, Successively iterative method for fractional differential equations with integral boundary conditions, Appl. Math. Lett., 38 (2014), 94-99. 1

[8] M. A. Krasnosel'skiĭ, Positive solutions of operator equations, Translated from the Russian by Richard E. Flaherty; edited by Leo F. Boron, Noordhoff Ltd. Groningen, (1964). 2

[9] S.-J. Li, X.-U. Zhang, Y.-H. Wu, L. Caccetta, Extremal solutions for p-Laplacian differential systems via iterative computation, Appl. Math. Lett., 26 (2013), 1151-1158. 1

[10] I. Podlubny, Fractional differential equations, An introduction to fractional derivatives, fractional differential equations, to methods of their solution and some of their applications, Mathematics in Science and Engineering, Academic Press, Inc., San Diego, CA, (1999). 1

[11] Y.-P. Sun, M. Zhao, Positive solutions for a class of fractional differential equations with integral boundary conditions, Appl. Math. Lett., 34 (2014), 17-21. 1, 1, 2

[12] C. Yang, C.-B. Zhai, Uniqueness of positive solutions for a fractional differential equation via a fixed point theorem of a sum operator, Electron. J. Differential Equations, 2012 (2012), 8 pages. 1

[13] C.-J. Yuan, Multiple positive solutions for $(\mathrm{n}-1,1)$-type semipositone conjugate boundary value problems of nonlinear fractional differential equations, Electron. J. Qual. Theory Differ. Equ., 2010 (2010), 12 pages. 2.5

[14] C.-B. Zhai, M.-R. Hao, Fixed point theorems for mixed monotone operators with perturbation and applications to fractional differential equation boundary value problems, Nonlinear Anal., 75 (2012), 2542-2551. 1

[15] C.-B. Zhai, L. Xu, Properties of positive solutions to a class of four-point boundary value problem of Caputo fractional differential equations with a parameter, Commun. Nonlinear Sci. Numer. Simul., 19 (2014), 2820-2827.

[16] C.-B. Zhai, W.-P. Yan, C. Yang, A sum operator method for the existence and uniqueness of positive solutions to RiemannLiouville fractional differential equation boundary value problems, Commun. Nonlinear Sci. Numer. Simul., 18 (2013), 858-66. 1

[17] X.-Q. Zhang, L. Wang, Q. Sun, Existence of positive solutions for a class of nonlinear fractional differential equations with integral boundary conditions and a parameter, Appl. Math. Comput., 226 (2014), 708-718. 1

[18] X.-K. Zhao, C.-W. Chai, W.-G. Ge, Existence and nonexistence results for a class of fractional boundary value problems, J. Appl. Math. Comput., 41 (2013), 17-31. 1, 2.4 